\title{
Optimized Ad-hoc On Demand Distance Vector Routing Protocol
}

\author{
Praveen Lalwani \\ PG, scholar, CSE Department \\ U.I.T, RGPV \\ Bhopal, India
}

\author{
Mahesh Motwani, PhD. \\ CSE Department \\ U.I.T, RGPV \\ Bhopal, India
}

\author{
Piyush Kumar Shukla \\ CSE Department \\ U.I.T, RGPV \\ Bhopal, India
}

\begin{abstract}
A Mobile Ad-hoc Network (MANET) is a collection of wireless mobile nodes forming a temporary network without using any centralized access point, infrastructure, or centralized administration. Data transmission between two nodes in MANET's may be requires multiple hops as the nodes transmission range is limited. Mobility of the different nodes makes the situation even more complicated. Multiple routing protocols especially for these conditions have been developed during the last few years, to find optimized routes from a source to some destination.

Ad-hoc network suffer from the lot of issues and congestion and security are the major issues which lead to severe degradation of network throughput and increases the routing overheads. This paper presents Optimized AODV which the modified version Enhance local repair AODV by improving its Route Error message format. The result shows that OAODV performs better in terms of routing overhead and end to end delay than AODV. The simulation is done through network simulator ns2 gives a detailed comparison of research on AODV. Open research direction is also discussed to serve as a starting point to protocol design and evaluation
\end{abstract}

\section{INTRODUCTION}

In AODV [1], the network [2] is silent until a communication is needed. At that point when any network node [3] wants to send the data to another node it needs to broadcast a route request packet for [4] a new connection [5]. Other AODV nodes forward this message, and when the route request message received at Target node then it replies by route reply packet. After the route establishment, communication takes place between the sender and receiver. If any of the link failure occurs in the communication path then route error packet is sent to the source. Unused entries in the routing tables are recycled after a time

When a link fails, a routing error is passed back to a transmitting node, and the process repeats

\section{LITERATURE REVIEW}

\subsection{AODV-BR (Backup Routing in Ad hoc Networks)}

This Research has been contributed by Sung-Ju Lee et-all [5]. This algorithm establishes the 'mesh and multipath without transmitting any extra control message. In this protocol the new style of routing called "on-demand" routing has been proposed for ad hoc networks.

\subsection{An Improvement on Ad-hoc Wireless Network Routing Based on AODV}

This Research has been contributed by Neda Moghim et-all [7]. In this paper try to reduce AODV's routing load by preventing AODV from relying on route request flood more often in the route discovery process. The simulation showed that the proposed way could reduce routing overhead and delay of routing in high load networks without any undesirable effects on throughput and delay of packets.

\subsection{A Robust Routing Protocol for Wireless Mobile Ad-Hoc Networks}

This Research has been contributed by Q. Wang et-all [8]. Further improvements are obtained by adapting Route Expiry Timeout (RET) to mobility at the expense of a slight increase in overhead. Future work may need to introduce mechanisms to enable traffic discrimination in networks with traffic of different priorities and characteristics including normal data. Voice, video, and web interaction traffic etc.

\subsection{AODV Multipath Extension Uses Source Route Lists with Optimized Route Establishment.}

This Research has been contributed by Yusuke Sakurai et-all [9] This paper introduces novel on-demand multipath routing protocol for MANET which combines the metrics of delay, hop count and disjointness, each intermediate node deliberately selects multipath candidates while contributing to suppression of unnecessary routing packets. Due to the extension of the RREQ / RREP packet provide more efficient multipath routes. The outcome of this research has higher packet delivery ratio and lower routing packets. Further research issue is to the improvement of the metric definition should be considered

\subsection{An Optimized AODV Protocol In Mobile Ad-hoc Network.}

This Research has been contributed by Zhao Qiang Zhu Hongbo et-all [12]. This paper is a new scheme to improve AODV protocol by the concept of reliable distance. The Ad Hoc on Demand Distance Vector (AODV) protocol is an ondemand protocol specialized for mobile ad hoc network due to node mobility and limited transmission range. 


\subsection{Throughput Enhancement in AODV Routing Using Mobility Awareness.}

This Research has been contributed by Dr. S. A. Hussain etall [11]. If a node in a mobile ad hoc network aware of the mobility of the neighbor nodes then highly mobile node is to avoid becoming a part of routes, this will greatly reduce new path discovery towards the destination. A node in the network is aware to its neighbor by the help of inquiries and reply to inquiries .These inquiries are based on the hellow message. The outcome of this research is greatly reducing the new path discoveries and it increases the network throughput and performance

\subsection{Performance Comparison of Routing Protocols for Mobile Ad-hoc Networks.}

This Research has been contributed by Vahid Nazari Talooki et-all [10]. In this research comparison of the AODV, DSR and TORA routing protocols is done with respect to modified path optimality.

\subsection{Performance Enhancement in AODV with Accessibility Prediction.}

This Research has been contributed by Habib-ur Rehman etall [3] In this paper a mechanism is introduced in which nodes are aware of its neighbor nodes. This information is utilized in routing operation like route request, route reply and route error operation. The outcome of this research it greatly reduces the routing overhead and MAC overheads.

\subsection{A Simulation Based Study of On- Demand Routing Protocols for Ad-hoc Wireless Networks.}

This Research has been contributed by Azzedine Boukerche et-all [6]. They have presented an extensive simulation studies to compare three Ad-hoc protocols DSR, AODV, and CBRP. In the Presence of different workload such as mobility, load and size of the Ad-hoc networks. Results indicate that the two source routing based protocols, DSR and CBRP, have very high throughputs while the distance-vector based protocol, AODV, exhibits a very short end-to-end delay of data packets.

\subsection{Performance Comparison and} Evaluation of AODV, OLSR, and SBR in Mobile Ad-Hoc Networks.

This Research has been contributed by Alexander Klein et-all [13]. . These protocols are compared on the basis of reliability and routing overhead. The outcome of this research shows SBR achieve high end to end reliability without frequent end to end route calculations. Future direction of research is to increase the reliability without increasing the routing overhead

\subsection{An Enhanced AODV for Mobile Ad- hoc Network.}

This Research has been contributed by HONG-PENG WANG et-all [14]. Enhance AODV protocol, is the enhance version of AODV in which mobile nodes aware of the local connectivity to its neighbor in the network it achieved by the extension of the hello message but with the lower overhead. It also prevents unidirectional link in the network to some extent
The outcome of this research is to reduce route load in the network. The s security of the protocol is another direction for our future work.

\subsection{ENHANCED AODV Routing Protocol for Ad hoc Networks.}

This Research has been contributed by Khushboo Agarwal etall [15]. EAODV is the next generation of AODV which offer quick adaptation to dynamic link condition, low processing overhead and low network utilization. EAODV reduces hop count, latency time and enhance throughput, packet delivery ratio in MANET. It also maintains multiple routes towards the destination and also the shortest route. Future direction of research covers the security aspect which is the major issue of research in MANET

\subsection{Enhanced Local Repair AODV (ELRAODV).}

This Research has been contributed by Jagpreet et-all [1,] The enhance local repair AODV is based on the local repair Strategy where unicast mechanism has been introduced to improve the routing overhead by making mobile nodes aware of local connectivity .The proposed Methodology extended the HELLO packet to NHellow. This extra information helps AODV to repair the route by unicast instead of broadcast. ELRAODV performs better than classic AODV in term of the congestion and end to end delay But the drawback of this mechanism is that it cannot work when multiple link failures. In Future this proposes protocol can be tasted on scalability and energy performance measures

\subsection{Performance Comparison of AODV, DSR and ZRP Routing Protocols In MANET'S}

This Research has been contributed by Shaily Mittal et-all [4]. This review paper has the aim of comparing various routing protocols and identifies drawbacks of each one of them. This research shows a comparison over different parameter like an average end to end delay, TTL based hop count and packet delivery ratio. In future research required to remove routing overheads of each one of them

\subsection{Enhanced AODV for Wireless Networks.}

This Research has been contributed by Sandeep Suman et-all [16]. E-AODV is the extensions of AODV in which two concepts are merging Blocking Expanding Ring Search (BERS) \& routing packets as HELLOW packet techniques for the reduction of routing overhead. The outcome of this research is that E-AODV performs better than AODV. Future research issues are Route Reply packet is a unicast packet which can also be used as a HELLO packet by utilizing promiscuous mode of a wireless node and HELLO load can be further reduced by using DATA packets as HELLO packets

\subsection{The Improved AODV Protocol with lower Route Cost and Smaller Delay (PWAODV).}

This Research has been contributed by Wang Ningning et-all [2]. In this piggyback mechanism and weighted neighbor stability algorithm is introduced. In this they were using a 
piggyback mechanism by which Route cost can be reduced greatly.

\section{PROPOSED Work to Decrease the Routing Overhead AND END TO END DELAY:}

Enhance local Repair AODV is motivated by the issues identified in local Repair AODV uses broadcast locally to repair the route. In ELRAODV route repair is done by unicast mechanism instead of broadcast. As shown in figure 1.1 when a blue node is down then instead of discarding the whole route or finding a new route from the source, Node 1 start repairing the same route to node 2 by finding the alternative of the broken node. As shown in the figure Node 1 sends a unicast request to node 2 by LRREQ Message \& Node 2 replies by RREP Message

Optimized AODV is modified version of ELRAODV .If the route is not repaired by ELRAODV then it sends the route error message towards the source with the help of RRER Packet to report the route failure or link failure. In our proposed research instead of sending RRER packet it sends PRRER (priority route error packet) which is the extension of RRER packet to set the priority of the packet. If some packet is waiting in the queue to be forwarded the proposed method instead of forwarding these packets, forwards the priority packet is first. As shown in the figure 1.1 if the route is not repaired by alternative purple node or multiple link failure occurs then it sends PRRER packets to the source

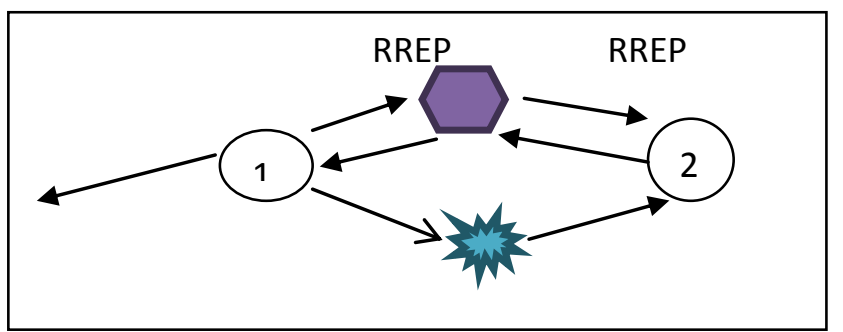

Fig 1.1 on planned AODV

\subsection{PROPOSED ALGORITHM}

Step 1: A RREQ message is broadcasted when a node needs to find out a route to a destination

Step 2: If RREQ Message is received then "The destination route is replying by unicasting a RREP Message back to the source route and also make an entry of next to next node in the Routing Table"

Step 3: If (Hello Timer Expire or Link Breaks)<smiles>[Tl]</smiles>

"Recover the Route by Next to Next Node" Else if "Recover the Route by Local Repair"
"Send PRRER (priority Route Error), Go to step 1" \}

Else "Send Data Packet"

\}

Step 4: End

\subsection{DESCRIPTION OF ALGORITHM}

3.2.1. A RREQ message is broadcasted when a node needs to find a new route to a destination. As a RREQ propagates through the network, intermediate nodes use it to bring up-todate their routing tables (in the direction of the source node). The RREQ also contains the most current sequence number for the destination. A valid destination route must have a sequence number at least as great as that enclosed in the RREQ

3.2.2 RREP messages are required when a RREQ Message reaches a destination node; the destination route is made available by unicasting a RREP back to the source route. A node generates a RREP if:

1. It is itself the destination.

2. It has a route to the destination. Ex: an intermediate node may also return with a RREP if it has a "fresh enough" route to the destination.

As the RREP circulates back to the source node, intermediate nodes update their routing tables (in the direction of the destination node). It makes two entries in the Routing Table one is for Next Node and for the node thereafter.

3.2.3 As we know all nodes are Mobile Nodes in the Ad-hoc Network. Communication failure occurs or link failure occurs because Node can move out of the network or it can go down due to energy limitation. In case of link failure it repairs the route by next to next node. As shown in figure1. 2 when a blue node goes down then instead of removing the whole route or to find a new route from the source, Node 1 start repairing the same route to node 2 by finding the alternative of the broken nose. As shown in the figure 1.2 Node 1 sends a unicast route repair request to node 2 by LRREQ Message \& Node 2 replies by RREP Message. In case of multiple link failures route cannot be repaired 


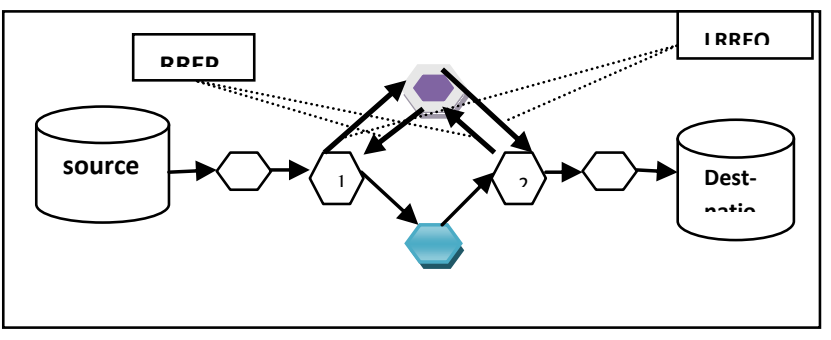

FIG 1.2

3.2.3.1 If Route is not restored from Next to Next Node it calls the Local Route repair mechanism of AODV by itself otherwise send the Priority Route Error message to the source as shown in figure 1.3

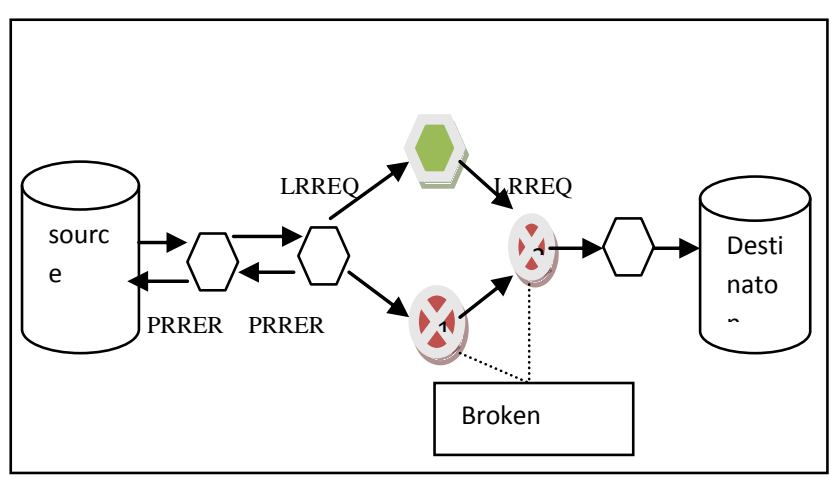

FIG1.3

3.2.4 PRERR (Priority Route Error) messages are generated when the link is broken. PRRER is generated by a node or passed on when received from another node. PRRER is the extension of RRER packet to set the priority of the packet. If some packets are waiting in queue keeping this packet aside PRRER packet is forward first. As shown in the figure 1.3 if the route is not repaired by alternative green node or multiple link failure occurs then it sends PRRER packets to the source. As we know source doesn't stop sending the data packet until gets the route error packet (PRRER). If it gets this packet it immediately stops sending data packets which effects on network overhead and congestion in the network

3.2.5 When PRRER Message is acknowledged as the source it again broadcast the RREQ Message to the network to set the new path and repeats the whole process again

\section{SIMULATION AND ANALYSIS:}

Table 1.1 The Parameters Taken as input are shown

\begin{tabular}{|c|c|}
\hline Transmitter Range & $250 \mathrm{~m}$ \\
\hline Model & Random Way Point \\
\hline Bandwidth & $2 \mathrm{Mbits} / \mathrm{s}$ \\
\hline Simulation Time & 100 \\
\hline Number of nodes & $10,20,30$ \\
\hline Scenario size & $600 \times 600 \mathrm{~m} 2$ \\
\hline Traffic type & Constant Bit Rate \\
\hline Packet size & 64 bytes \\
\hline Rate & 20 packets/s \\
\hline
\end{tabular}

TABLE1.1: Inputs
Simulation has been made on 1020 and 30 Nodes as shown in the table 1.1, There are two parameters tested here one is end to end delay and another one is routing overhead, These parameters are directly related to congestion if congestion is reduced then these two parameters will also reduce. Both of the parameters get reduced as shown in the Table 1.2 and Table 1.3 and graph of experimental result in fig 1.4 and fig 1.5. Simulation has been done on $\mathrm{ns} 2$

Table 1.2 comparisons of AODV and OAODV on ETE

\begin{tabular}{|l|l|l|}
\hline $\begin{array}{l}\text { No. of } \\
\text { Nodes }\end{array}$ & $\begin{array}{c}\text { End to End Delay of } \\
\text { AODV }\end{array}$ & $\begin{array}{c}\text { End to End Delay of } \\
\text { OAODV }\end{array}$ \\
\hline 10 & 12.01 & 10.01 \\
\hline 20 & 560 & 112.12 \\
\hline 30 & 225 & 41 \\
\hline
\end{tabular}

Table 1.3 comparisons of AODV and OAODV on RO

\begin{tabular}{|l|l|l|}
\hline $\begin{array}{l}\text { No. of } \\
\text { Nodes }\end{array}$ & $\begin{array}{l}\text { Routing Overhead } \\
\text { of AODV (ms) }\end{array}$ & $\begin{array}{l}\text { Routing Overhead of } \\
\text { OAODV (ms) }\end{array}$ \\
\hline 10 & 8.0 & 12.5 \\
\hline 20 & 57.01 & 45 \\
\hline 30 & 100.01 & 70 \\
\hline
\end{tabular}

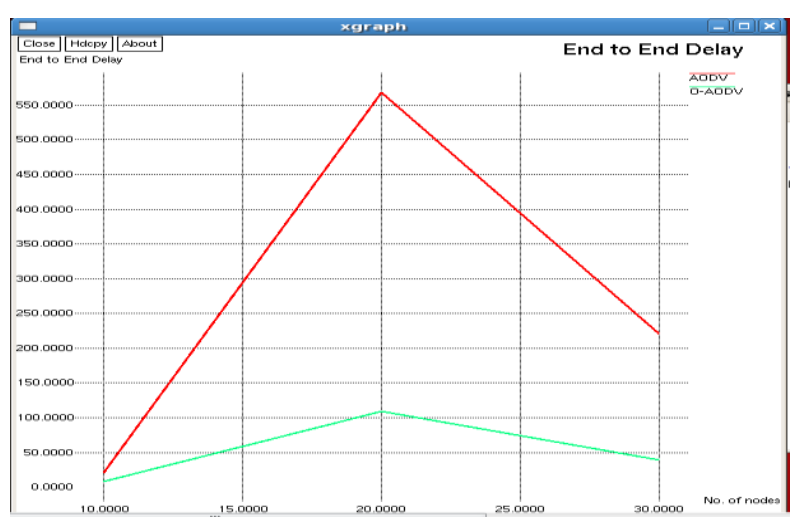

Fig 1.4

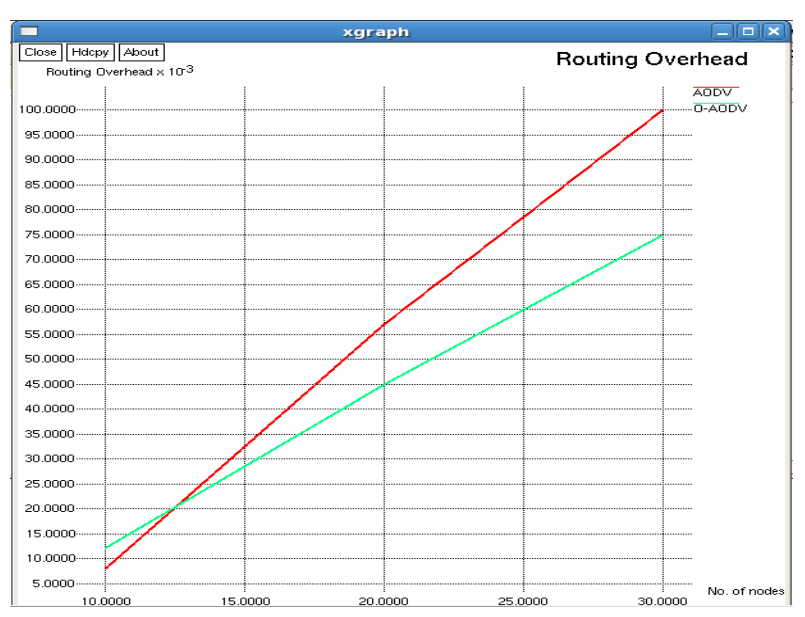

Fig 1.5 


\section{CONCLUSION AND FUTURE RESEARCH DIRECTION}

In this paper we have done surveys of the papers published on AODV and simulation of the proposed Optimized AODV The results as shown in fig 1.4 clearly show that as the number of nodes increase routing overhead decreases in OAODV as compared to the AODV. Due to the lower routing overhead of OAODV improves the network performance. The results shown in fig 1.5 indicate as the number of nodes increase end to end delay decreases in OAODV as compared to the AODV.

The proposed solution is in most cases not tested in a real environment Therefore, future studies should rather be devoted to real implementation than just a simulation. Only such an approach can ultimately verify a protocol's usefulness in future Ad-hoc network

\section{REFERENCES}

[1]. P. Singh, S. Rani, "Enhanced Local Repair AODV" International Conference on Advances in Computing, Control, and Telecommunication Technologies 12 Jan 2010 pp. 787-2010.

[2]. W. Ningning, C. Yewen, "Improved AODV protocol with Lower Route Cost and Smaller Delay" IEEE Fourth International Conference on Intelligent Computation Technology and Automation 15 April 2011 pp.7-11.

[3]. H. Rehman, L. Wolf, "Performance Enhancement in AODV with Accessibility Prediction" IEEE International conference on sensor network 12 Jan 2008 pp. 1-6.

[4]. S. Mittal, P. Kaur "Performance Comparison Of AODV, DSR and ZRP Routing Protocols In MANET'S" IEEE International conference on Advances in computing, control and Telecommunication 12 Jan 2010 pp. 165169.

[5]. S.J. Lee and M. Gerla "AODV-BR: Backup Routing in Ad hoc Networks", IEEE, Jan 2000, pp. 1311-1316.

[6]. A. Boukerche, "A Simulation Based Study of On-Demand Routing Protocols for Ad hoc Wireless Networks" IEEE, Jan 2008, pp. 85-93.
[7]. N. Moghim "An Improvement On AD-HOC Wireless Network Routing Based On AODV", The 8th International Conference Communication Systems, 2002. ICCS 2002 25-28 Nov. 2002, pp. 1068 - 1070 vol.2

[8]. Q. Wang "A Robust Routing Protocol For Wireless Mobile Ad-Hoc Networks", The 8th International Conference on Communication Systems, 25-28Nov 2002, pp. 1071 - 1075 vol.2.

[9]. Yusuke "AODV Multipath Extension uses Source Route Lists with Optimized Route Establishment", 2004 International Workshop on Wireless Ad-Hoc Networks, 31 May-3 June 2004, pp. 63 - 67

[10]. V.N. Talooki, "Performance Comparison of Routing Protocols For Mobile Ad Hoc Networks", Asia-Pacific Conference on Communications, 2006. APCC'06. Aug. 31 2006-Sept. 1 2006, pp. 1 - 5

[11]. S.A. Hussain, E. Garcia, M. Idrees, "Throughput Enhancement in AODV Routing Using Mobility Awareness", 9th International Multitopic Conference, IEEE INMIC 2005, July 2005, pp. 1-4.

[12]. Z. Qiang, Z. Hongbo "An optimized AODV protocol in mobile ad hoc Network”, IEEE, April 2004, pp. 1-4.

[13]. A. Klein, "Performance Comparison and Evaluation of AODV, OLSR, and SBR in Mobile Ad-Hoc Networks", IEEE ISWPC 2008, Jan 2008, pp. 571-575.

[14]. H.P. Wang, L. Cui “ An Enhanced AODV for Mobile Adhoc Network" , Proceedings of the Seventh International Conference on Machine Learning and Cybernetics, Kunming, 12-15 July 2008.

[15]. K. Agarwal, Dr. L.K. Awasthi "Enhanced AODV Routing Protocol for Ad hoc Networks", ICON IEEE, 2008, pp. 1-5.

[16]. S. Suman and Balkrishan "Enhanced AODV for Wireless Networks", IEEE 2nd International Advance Computing Conference, 2010, pp. 246-250.

[17]. C.E. Perkins, E.M. Belding-Royer, and S.R. Das, "Ad hoc On-Demand Distance Vector (AODV) Routing", IETF Experimental RFC 3561, Jul. 2003 